\title{
Basic Requirements for Monitoring Sedated Patients: Blood Pressure, Pulse Oximetry, and EKG
}

\author{
Walter G. Maurer ${ }^{a}$ Michael Walsh ${ }^{b} \quad$ Nikos Viazis ${ }^{c}$ \\ ${ }^{a}$ Ambulatory Anesthesia, Department of Anesthesia, Anesthesia Institute, Cleveland Clinic, Cleveland, Ohio, and \\ ${ }^{b}$ Mayo Medical School, Mayo Clinic, Rochester, Minn., USA; ${ }^{c} 2$ nd Department of Gastroenterology, Evangelismos \\ Hospital, Athens, Greece
}

\section{Key Words}

Non-anesthesia providers · Pulse oximetry • EKG

\begin{abstract}
The American Society of Anesthesiologists published monitoring requirements for non-anesthesia providers performing sedation and analgesia in 2002. This manuscript covered not only the monitoring of patients under sedation and analgesia but pre-procedure evaluation and preparation, personnel availability and training, emergency equipment availability, use of supplemental oxygen, sedative-analgesic agents, and discharge criteria. Current recommendations for monitoring include patient responsiveness, blood pressure, respiratory rate, heart rate, and oxygen saturation. Oxygen saturation is a critical vital sign, but there can be a significant delay between inadequate ventilation and desaturation. Supplemental oxygen can dangerously increase this disconnect. Thus, one must monitor adequacy of ventilation by direct observation, auscultation, and/or end-tidal $\mathrm{CO}_{2}$ monitoring. The most important admonition is that 'ventilation' and 'oxygenation' are not the same.
\end{abstract}

Copyright $\odot 2010$ S. Karger AG, Basel

\section{Introduction}

In 1995, the American Society of Anesthesiologists (ASA) published a review of basic requirements for nonanesthesia providers performing sedation and analgesia. In 2002 [1], revision of these ASA practice guidelines was published again in the journal Anesthesiology. These practice guidelines came out of the ASA Committee on Practice Parameters using a very rigorous approach, and were produced by nine anesthesiologists working with a gastroenterologist, Dr. Gregory Zuccaro. They reviewed the literature covering 3,000 citations which yielded 357 articles with direct-linkage-related evidence. The committee followed this with a broad multispecialty survey of consultants, then a consensus process, followed by open forums, and finally formal approval from the ASA House of Delegates. The ASA definitions are as follows:

- Minimal sedation: normal response to verbal commands; cognitive function and coordination may be impaired; airway, ventilation, and cardiovascular functions are unaffected.

- Moderate sedation: purposeful response (not reflex withdrawal from painful stimulus) to verbal or tactile stimulation; no airway intervention; adequate spontaneous ventilation; cardiovascular function is usually maintained.

Walter G. Maurer, MD

Anesthesia Institute, Department of General Anesthesia, Desk E3

9500 Euclid Avenue

Cleveland, OH 44195 (USA)

Tel. +1 216444 1192, Fax +1 216444 9247, E-Mail MAURERW@ccf.org 
- Deep sedation: purposeful response after repeated or painful stimulation; airway intervention may be required; spontaneous ventilation may be inadequate; cardiovascular function is usually maintained.

- General anesthesia: unarousable even with painful stimulus; airway intervention often required; positive pressure ventilation may be required; cardiovascular function may be impaired.

This manuscript covered not only the monitoring of patients under sedation and analgesia, but also pre-procedure evaluation and preparation, personnel availability and training, emergency equipment availability, the use of supplemental oxygen, sedative-analgesic agents and IV access, and recovery and discharge criteria.

\section{Causes of Morbidity during Sedation}

Both the anesthesia literature and the gastrointestinal literature conclude that the primary causes of morbidity during sedation and analgesia are respiratory depression and airway obstruction. A recent ASA closed claims study on monitored anesthesia care in non-operating room locations reaffirmed this finding, but also noted respiratory events were twice as likely to cause morbidity in 'off-site' locations compared to the operating room [2]. The vast majority of incidents in this study took place in gastrointestinal suites (82\%). A recent review of the gastrointestinal Clinical Outcomes Research Initiative (CORI) database [3] also found that cardiopulmonary events were the leading cause of unplanned events during gastrointestinal endoscopy.

Risk factors included patient's age, higher ASA status, inpatients, trainee participation, and routine use of oxygen. Most of these emphasize the importance of preoperative evaluation and proper triage, but the routine use of oxygen deserves special attention and will be discussed below.

\section{How to Monitor Respiration Rate}

Methods to monitor respiration include: direct observation, auscultation, capnography, and/or EKG analysis of respiratory rate via impedance pneumography. It is obviously very difficult to monitor breathing under direct observation in a dark gastrointestinal suite with limited 'hands on' access to the patient. While direct visualization of chest movement and/or impedance pneumography can be useful, neither detects actual airflow at the oropharynx. To rule out obstruction and actually monitor ventilation, auscultation and end-tidal $\mathrm{CO}_{2}$ monitoring are far more accurate. Vargo's group [4] reported 54 episodes of apnea/disordered breathing in 28 patients (49 total patients in the study), all of which were documented by end-tidal $\mathrm{CO}_{2}$ monitoring. Blinded observers detected only $50 \%$ of these by pulse oximetry, and none by direct visual assessment.

Keeping a contemporaneous record of the respiratory rate is important to monitor any adverse sedation trend (along with blood pressure, heart rate and oxygen saturation). It is also important to document in the sedation record any manipulation of the airway such as a chin lift, jaw thrust, or placement of nasal or oral airway. More intensive interventions include positive pressure mask ventilation, the use of an LMA (laryngeal mask airway), or ultimately the need for endotracheal intubation. If a gastroenterology provider is considering an LMA or other invasive airway device, he/she should immediately call for anesthesia support since further manipulation of the airway can lead to excessive secretions or bleeding, and result in a 'can't intubate/can't ventilate' situation (even for an experienced anesthesiologist).

\section{Ventilation versus Oxygenation}

One of the most important monitors is pulse oximetry and the tone generated by the waveform must be loud enough to be heard. On most monitors, the pitch of the waveform tone decreases as saturation decreases. Keep in mind, however, that there is a delay (monitor lag) of up to $1 \mathrm{~min}$ between the actual patient saturation and the digital readout on the saturation monitor (as well as the alarm). The physiologic lag time may be far greater, especially when supplemental oxygen is used; case reports of profound hypercarbia and acidosis, despite adequate oxygen saturation, have been reported [5]. The CORI database report (outlined above) found routine supplemental oxygen was a risk factor for cardiopulmonary events. The most important admonition is that 'ventilation' and 'oxygenation' are very different. Thus, a patient with good oxygenation is not always ventilating adequately. There is much debate as to whether all sedated patients should routinely receive supplemental oxygen [6-8]. While supplemental oxygen will decrease the overall incidence of desaturation, it can delay the recognition of significant airway obstruction and hypoventilation, in some cases beyond the ability to rescue. In an article published several years ago [9], in over 1,000 patients receiving flexible 
bronchoscopy, supplemental oxygen was only needed in $14 \%$ of the cases. The majority of patients did not require oxygen supplementation, especially the group with an $\mathrm{FEV}_{1}$ above 1 liter.

In summary, monitoring oxygenation by pulse oximetry is not a substitute for monitoring ventilation. This axiom is even more important as the depth of sedation increases.

\section{Importance of EKG and Blood Pressure Monitoring}

The EKG should be continuously monitored for arrhythmias (possible sign of acidosis and hypercarbia) and ischemia. It is obviously important to review the patient's underlying rhythm pre-procedure (bradycardia, atrial fibrillation, pacemaker). This will facilitate interpretation of any changes in the patient's rhythm under sedation. A careful pre-procedure medication history, including antiarrhythmic drugs, should be noted. If the patient has an AICD (automatic implantable cardiac defibrillator), one must be concerned over the use of electrocautery, as this can trigger the AICD inappropriately. This will occur whether the electrocautery is a bipolar or unipolar device. AICD patients should have the defibrillator turned off and external defibrillator pads placed if electrocautery is to be used. Personnel familiar with the device should be immediately available. In the sedation environment, one must remember that many arrhythmias are caused by hypercarbia and, in fact, pulseless electrical activity does result from hypoxia and acidosis (caused by hypoventilation and hypercarbia). Availability of advanced cardiac life support-trained personnel is essential.

When monitoring blood pressure, one must remember that this is not a 'continuous' patient monitoring as for respiratory rate, heart rate, and oxygen saturation. Blood pressure is usually taken every $5-15 \mathrm{~min}$. Thus, using blood pressure changes to determine the depth of sedation is challenging. Even with automated blood pressure monitoring every $5 \mathrm{~min}$, titration of sedation based on the blood pressure is difficult. One must also have knowledge of the baseline blood pressure and whether the patient has taken any antihypertensives prior to the procedure. Narcotics tend to block hypertension from light sedation. It is also important to remember that many sedation drugs can be synergistic with antihypertensives, especially ACE inhibitors.

In conclusion, the monitoring of patients under sedation must be closely addressed by the gastroenterologist in addition to the technique of the procedure itself. This requires the proper training of those in the room assigned to monitor the patient as well as excellent monitoring equipment.

\section{References}

Basic Requirements for Monitoring Sedated Patients
American Society of Anesthesiologists Task Force on Sedation and Analgesia by NonAnesthesiologists: practice guidelines for sedation and analgesia by non-anesthesiologists. Anesthesiology 2002;96:1004-1017.

-2 Sharma VK, Nguyen DD, Crowell MD, et al: A national study of cardiopulmonary unplanned events after GI endoscopy. Gastrointest Endosc 2007;66:27-33.

-3 Metzner J, Posner KL, Domino KB: The risk and safety of anesthesia at remote locations: the US closed claims analysis. Curr Opin Anaesthesiol 2009;22:502-508.

-4 Vargo JJ, Zuccaro G, Dumot JA, et al: Automated graphic assessment of respiratory activity is superior to pulse oximetry and visual assessment for the detection of early respiratory depression during therapeutic upper endoscopy. Gastrointest Endosc 2002; 55:826-831.
5 Hutton P, Clutton-Brock T: The benefits and pitfalls of pulse oximetry. BMJ 1993;307: 457-458.

6 Fu ES, Downs JB, Schweiger JW, Miguel RV, Smith RA: Supplemental oxygen impairs detection of hypoventilation by pulse oximetry. Chest 2004;126:1552-1558.

$\checkmark 7$ Davidson JA, Hosie HE: Limitations of pulse oximetry: respiratory insufficiency - a failure of detection. BMJ 1993;307:372-373.

$\checkmark 8$ Deitch K, Chudnofsky DR, Dominici P: The utility of supplemental oxygen during emergency department procedural sedation with propofol: a randomized controlled trial. Ann Emerg Med 2008;52:1-8.

- Jones AM, O’Driscoll R: Do all patients require supplemental oxygen during flexible bronchoscopy? Chest 2001;119:1906-1909. 